\title{
Effect of Amputation Level on the Stress Transferred to the Femur by an Artificial Limb Directly Attached to the Bone
}

L. Newcombe ${ }^{1,2}$, M. Dewar ${ }^{2}$, G. Blunn ${ }^{1}$, P. Fromme ${ }^{2}$

${ }^{1}$ UCL Institute of Biomedical Engineering (IBME), John Scales Centre for Biomedical Engineering, Institute of Orthopaedics and Musculoskeletal Science, University College London, Royal National Orthopaedic Hospital, Brockley Hill, Stanmore, Middlesex, HA7 4LP, UK

${ }^{2}$ UCL Institute of Biomedical Engineering (IBME), Department of Mechanical Engineering, University College London, London, WC1E 7JE, UK

\begin{abstract}
Attachment of an artificial limb directly to the skeleton has a number of potential benefits and the technique has been implemented for several amputation sites. In this paper the transfer of stress from an external, transfemoral prosthesis to the femur during normal walking activity is investigated. The stress distribution in the femur and at the implant-bone interface is calculated using finite element analysis for the 3D geometry and inhomogeneous, anisotropic material properties obtained from a CT scan of a healthy femur. Attachment of the prosthetic leg at three different levels of amputation is considered. Stress concentrations are found the distal end of the bone and adjacent to the implant tip and stress shielding is observed adjacent to the implant. It is found that the stress distribution in the femur distal to the epiphysis, where the femur geometry is close to cylindrical, can be predicted from a cylindrical finite element model, using the correct choice of bone diameter as measured from a radiograph. Proximal to the lesser trochanter the stress decreases as the femur geometry diverges significantly from a cylinder. The stress concentration at the distal, resected end of the bone is removed when a collared implant is employed. These findings form the basis for appropriate settings of an external fail-safe device to protect the bone from excessive stress in the event of an undue load.
\end{abstract}

\section{Introduction}

Skin problems on the stump are common in transfemoral amputees ${ }^{1}$. Attaching the prosthesis directly to the skeleton removes the need for applying loads across the skin. Possible benefits of direct skeletal attachment of a prosthetic leg include the elimination of skin associated problems and blood occlusion from a rubbing socket. Furthermore, the hip joint is not obstructed by a socket, allowing free movement ${ }^{2}$, and better control of the artificial limb is possible, as the forces and moments are not transmitted through a cushion of soft tissue. The first systems to explore the possibility of percutaneous prosthetic implants were dental implants ${ }^{3,4}$. The attachment of bone to the anchor is regarded as the reason for long-term success of dental implants ${ }^{5}$.

A number of trans-femoral designs are currently in use and the technology has also been applied to arm, finger and maxillofacial prostheses. At the University of Lübeck a design called the 'ISP 
Endo-Exo Prosthesis' is being developed in association with ESKA GmbH in Lübeck, Germany, with the first prosthesis implanted in $1999^{6}$. The current design with the longest clinical experience has been developed at the University of Gothenburg, Sweden, in association with Integrum $A B$, Gothenburg$^{2}$. The rehabilitation programme for this, devised in 1990, has been given the acronym OPRA (Osseointegrated Prosthesis for the Rehabilitation of Amputees) ${ }^{7}$.

Finite element analysis (FEA) has been used for analysing the stresses in and around the complex geometries of orthopaedic implants ${ }^{8-11}$. In order to develop safety features and protect the bone from dangerous loads it is important to know the stress distribution, concentration and shielding around the implant for the attachment of a transfemoral prosthesis. Geometry and loading of the femur vary for an amputee with different levels of bone resection, as investigated by Zheng et al. ${ }^{12}$. For the geometry of the Brånemark OPRA implant, which is threaded and screwed into the bone, several FEA studies of the implanted femur have been published. Using a simplified axisymmetric model of the bone and implant, $\mathrm{Xu}$ et al. ${ }^{13}$ found stress concentrations in the bone near the implant tip and at the end of the bone. In a further study Xu et al. ${ }^{14}$ used data from a CT scan to model the OPRA implant in-situ, taking the screw thread into account. An implant with a smaller diameter was found to cause less stress shielding in the bone because the narrower implant is more flexible. High stress at the end of the bone, and an increase in peri-implant stress as the level of amputation became more proximal was predicted. The load transfer for both the ISP Endo/Exo prosthesis and the OPRA system was calculated and compared to the stress and strain energy density an intact femur to predict bone failure and bone remodelling ${ }^{15}$. The risk of failure of an osseointegrated fixation was investigated for both a simplified and patient-specific FEA model and found to increase significantly ${ }^{16}$. A novel design for the intramedullary fixation has been proposed to reduce stress shielding and risk of failure ${ }^{17}$.

This contribution documents an investigation into a new prosthetic attachment system which is undergoing clinical trials and has been given the acronym 'ITAP': 'Intraosseous Transcutaneous Amputation Prosthesis'. The system comprises a cylindrical stem which is press-fit into the intramedullary cavity of the femur and HA coated to encourage osseointegration of the implant. The implant protrudes through the skin, providing an anchorage point for the attachment of the prosthetic limb. A technique has been devised to combat infection by encouraging skin epithelial cells to attach to the metal implant, creating a seal against infection ${ }^{18}$. The prosthetic limb includes a patented fail-safe device which decouples the prosthesis from the implant when the loading of the prosthesis exceeds the safe load for the bone, with independently variable activation loads for the torsional and bending moments. In this contribution the stress distribution in the femur has been calculated for three anatomical FEA models with different levels of bone resection to inform the choice of the activation loads for the fail-safe device. Results from the anatomical FEA models based on the CT scan of a healthy femur were compared with homogeneous, cylindrical FEA models to assess a simpler option to predict stress levels for a patient. 


\section{Materials and methods}

\subsection{Creation of finite element models of resected femurs from CT}

A human cadaveric femur was scanned every two millimetres from the femoral head to the lesser trochanter and then at five millimetre intervals through the rest of the bone. The CT images were saved in JPEG format and imported into MIMICS 9.1. The information was used to create a threedimensional image of the bone. Superfluous attached soft tissue was removed by erasing it from each of the images to leave the outline of the bone. A surface mesh of the femur was then created. The surface mesh was exported in PAT format and used to create volume meshes in MARC/MENTAT 2005 (MSC Software, USA). Hexahedral elements were used that had $1 \mathrm{~mm}$ edge length at the bone-implant interface and the FEA models were checked for convergence.

\subsection{Materials for FEA Model}

The volume mesh models of the femur were exported to MIMICS for the allocation of the inhomogeneous and orthotropic material properties (Fig. 1). For each element the material properties were assigned based on the average density in that volume of the CT scan. Taddei et al. ${ }^{19}$ compared different mapping techniques from the Hounsfield Unit (HU) to the Young's moduli and validated the FEA results with an experimental measurement. Using a voxel-based FEA model Keyak et al. ${ }^{20}$ predicted the patient-specific strength of femurs tested to failure and found good correlation. The grey values were converted into five material types using methods described by Taylor et al. ${ }^{21}$ and Rho et al. ${ }^{22}$. Previous researchers using FEA have divided bone into regions of different material alignment and produced realistic deformations when compared with a mechanical model ${ }^{23}$. This method was employed and the femur was divided into regions of common orthotropic alignment. In the diaphysis the material properties are aligned with the axis of the bone, while at the proximal end the alignment varies approximately with the direction of loading $^{24}$. The total material spectrum was divided into five different sections as specified in Table 1. The implant model was composed of titanium alloy (Ti6Al4V) and had a Young's modulus of 110GPa and Poisson's ratio of 0.3 .

\subsection{Levels of amputation}

Three levels of bone resection were modelled: the three models represent a long residual limb (one quarter of the bone removed), a short residual limb (three-quarters of the bone length removed) and a resection level between the two (half of the bone removed). A different implant was designed for each level of resection and the meshes were formed to fit around an implant, with a hollow to match the shape of the implant. Based on experience of designing intramedullary implants the stems were designed for maximum cortical bone contact. The outline of the implant shapes and the dimensions are shown in Fig. 1. For the $3 / 4$ resection level a larger implant diameter (18mm instead of $14 \mathrm{~mm}$ ) was used, and the implant contact length was only $50 \mathrm{~mm}$ as compared to $160 \mathrm{~mm}$ and $140 \mathrm{~mm}$ respectively for the lower resection levels. 


\subsection{Alignment of implant}

In Fig. 1a the cavity for the implant for the longest residual limb has cortical bone adjacent to most of its length. At the distal end of the bone, there is little cortical bone contact because the cortical bone diverges towards the knee. Fig. 1b shows the femur with half of the femur resected. The contact of the implant with the cortical bone is patchy along most of the length of the implant and reduces near the proximal end. The implant is in an area of cancellous bone at the proximal end, which lies inside the widened diameter of the lesser trochanter. Fig. 1c shows how a 5 degree bend in the implant is required to take account of the natural curvature of the femur. Fig. 1d shows the three-quarters resected femur, where the larger diameter implant is only partially in contact with cortical bone. Perfect contact conditions were used for all models, representing good bone integration (osseointegration) with the implant, similar to previous studies ${ }^{9,13}$.

\subsection{Analysis of the models}

The models of the bone were exported back into MARC/MENTAT, coupled with the implant meshes, and analysed. The implant meshes were extended to the level of the knee, and bending force, torsional moment and axial force were applied at the level of the knee. The bone was constrained to be rigidly held at the femoral head and at the greater trochanter, where the femur is restricted in movement by bony contact and muscle respectively. No muscle loads were applied along the length of the bone, as the position and strength of the muscles attached to the femur of the transfemoral amputee vary and differ widely in individual amputees and from the nonamputee. Excluding the muscle loads along the length of the bone represents the highest load case.

The loads were obtained from a study in which a load-cell had been incorporated into osseointegrated prostheses to measure the forces and moments in the external prostheses for twelve amputees during walking ${ }^{25}$. The loads applied to the FEA were the maximum experienced during normal daily activities. The data were given in percentage of bodyweight and have been converted to be relevant for a patient with a weight of $750 \mathrm{~N}$. Similar studies ${ }^{26,27}$ have been performed to measure loads for femoral amputees. The applied loads were $143 \mathrm{~N}$ lateral load applied at the position of the knee to generate shear forces and bending moments, $664 \mathrm{~N}$ axial load and $8 \mathrm{Nm}$ axial torque. The loads were applied individually as three different loading conditions, so that each type of load could be investigated separately. The models were linear so the stresses calculated could be summed to obtain the stresses experienced under combined loading.

\subsection{Cylindrical finite element models}

For the three resection lengths cylindrical finite element models of comparable dimensions were created in MARC/MENTAT and constrained and loaded as described above. The dimensions and material properties of the implant were the same as for the anatomical models. The bone was modelled as cylindrical with various outer diameters and the inner diameter taken to be that of the 
implant (Fig. 2). The material properties of the bone were chosen as transversely isotropic with Young's modulus of 20GPa longitudinally and 12GPa transversely and Poisson's ratio of 0.38 to match those of the cortical bone in the anatomical models.

Using the cylinder simplification to assess the stresses in the femur of an amputee with ITAP, there are different ways to choose the outer diameter for a cylindrical representation of the bone shaft. Three are considered here: (a) by matching the cross-sectional area of the bone, (b) by matching the broadest or narrowest part of the bone cross-section using the distance from the centre of the intramedullary canal to the outer surface of the bone described as the 'radius' by Stephenson and Seedhom ${ }^{28}$, or (c) by measuring the bone size in the A-P plane and the M-L plane and using the most appropriate of these measurements. Table 2 shows the cylinder bone diameters calculated from each of the three methods for the scanned femur at a cross-section half-way along the implant length. The diameters calculated in table 2 are in the range of $26 \mathrm{~mm}$ to $36 \mathrm{~mm}$. The smallest measured diameter of $26 \mathrm{~mm}$ was chosen for the comparison to the anatomical models.

\section{Results}

For typical walking loads the highest stresses were recorded in a cylindrical coordinate system along a line adjacent to the implant and a line on the outer surface of the bone. For the lower resection levels ( $1 / 4$ and $1 / 2$ bone removed) only the relevant normal and shear stresses for the different loading conditions are shown, i.e., the axial stress and the axial-radial shear stress in bending and axial loading, radial-tangential and the tangential-axial shear stresses in torsion. The maximum values of other stresses were less than one third of the stresses shown. For the highest resection level all stress components are shown.

\subsection{Stress distribution along implant for a bone with quarter of its length removed}

The stress distribution in the femur with one quarter of the bone removed was calculated using the anatomical model. As the geometry of the femur over the implanted length is close to cylindrical, for bending loads the axial and shear stresses in this anatomical model match those in the cylindrical model (26 mm bone diameter) on the stem/bone interface. There is no shear stress along the stem/bone interface (Fig. 3a) and outer surface of the bone (Fig. 3b), except for stress concentrations at the distal end of the bone and adjacent to the implant tip, which are less than $5 \mathrm{MPa}$. The axial stress recorded on the outer surface of the bone in bending is slightly lower and more variable for the anatomical model than for the cylindrical model, partially due to the irregular surface of the anatomical model and difficulty in choosing corresponding nodes. A linear increase in stress along the bone adjacent to the implant is caused by the increasing bending moment due to the lever-arm increasing from the point of load application. The stress level along the implant length is lower than for the femur alone due to the increased second moment of area of the femur and implant, introducing stress shielding. Significant stress concentrations at both the distal and proximal end of the bone (factor approximately 5 ) are predicted from both models. At the proximal 
end of the implant under bending load the highest axial stress of $22 \mathrm{MPa}$ is calculated. The von Mises stress was calculated to take the combined effect of all stress components into account, finding values of $35 \mathrm{MPa}$ at the implant tip and $17 \mathrm{MPa}$ at the distal end of the bone.

For axial loading the axial stress is lower adjacent to the implant than proximal to it (stress shielding), and there is a stress concentration with factor 2 at the end of the bone and factor 1.5 at the implant tip (Fig. 3c). Under typical walking loads the stresses due to axial loading are only about $10-20 \%$ of those found due to bending (Fig. 3c,d). The radial-tangential shear stress in torsion follows a similar pattern to the axial stress in axial loading for the cylindrical model. The most significant stress concentration occurs at the distal end of the bone with a high level of radial-tangential shear stress $(10 \mathrm{MPa})$. However, the stress along the length of the implant in the anatomical model (Fig. 3e) is approximately double the value found for the cylindrical model (diameter $26 \mathrm{~mm}$ ), as the anatomical model is not rotationally symmetric. Similar patterns are seen on the outer bone surface, but no stress concentration occurs (Fig. 3f).

\subsection{Cylinder model approximation}

The cylindrical model gives similar stress values to those found in the anatomical FE model under bending and axial load as long as the complete implant length is located in the shaft of the bone (implant tip distal to lesser trochanter). This indicates that the cylindrical model could be employed for assessment of the stresses occurring in the femur of an amputee with a long residual limb using ITAP. The diameters calculated in table 2 are in the range of $26 \mathrm{~mm}$ to $36 \mathrm{~mm}$, and the model with an outer bone diameter of $26 \mathrm{~mm}$ was found to correlate best with the anatomical model under bending and axial load. It appears that taking the linea aspera into account leads to an overestimation of the required bone diameter. 'Minimum radius' and ' $M-L$ size' measurements give cylinder diameters of 26.0 and $26.4 \mathrm{~mm}$ respectively. Therefore both are appropriate to select the cylinder diameter. In order to measure the minimum radius of the bone a CT scan is necessary for the amputee, while only a radiograph taken in the correct orientation is required to measure the M-L size of the bone. It is, therefore, proposed that this method be used to choose the diameter for a simple representation of the bone.

For torsional load the cylindrical model geometry with a diameter of $26 \mathrm{~mm}$ does not represent the stress in the bone adjacent to the implant well, where the bone-implant interface is believed to be most at-risk from overloading in torsion, especially during the period of bone healing after implantation $^{29}$. A further cylindrical model in torsion was created to match the stresses in the bone next to the implant. The geometry chosen has a $10 \%$ smaller outer diameter of $23 \mathrm{~mm}$. Fig. 4 shows the shear stress recorded in this narrower cylinder model matches the anatomical model significantly better than the $26 \mathrm{~mm}$ diameter cylindrical model (Fig. 3e,f). This is assumed to be due to the irregular shape of the bone cross-section and has to be treated separately from the bending and axial load cases. 


\subsection{Collared Implant}

One possible way to remove the stress concentration at the distal end of the bone is to include a collar in the design of the implant. Figure 5 shows the axial stress at the stem/bone both with and without a collar. The collar is assumed to be osseointegrated, and the stress at the distal end of the bone is distributed across the resected surface of the bone, removing the stress concentration the stem/bone interface. Similar results were found in torsion and axial loading where the stress concentration at the distal end of the stem/bone interface was removed by the addition of a collar.

\subsection{Stress distribution along implant for a bone with half of its length removed}

The stress in the bone with half of the length removed is shown in Fig. 6. For an axial (Fig. 6c,d) and torsional (Fig. 6e,f) load the predicted stress levels along the implant are comparable to the model with quarter of the femur resected (Fig. 3). In bending (Fig. 6a,b) the stress adjacent to the implant is greater than the interface stress for the femur with one quarter removed due to the longer lever-arm. The stresses follow the pattern of the cylindrical model on the inner surface of the bone until the geometry of the femur causes a reduction in stress. There is an increase in the cross-sectional area of the bone due to the lesser trochanter, which significantly affects the second moment of area and polar moment of area of the bone. This coincides with the proximal end of the implant, resulting in the stress in the anatomical model being lower than the stress predicted by the cylindrical model. The cylinder approximation indicates that a high stress concentration (65MPa in bending, Fig. 6a) would be evident if the implant tip were in the diaphysis. The stresses on the outside of the bone are diminished to the same extent as the stress on the inside of the bone by the geometry of the proximal femur in all three loading conditions.

\subsection{Stress distribution along implant for a bone with three quarters of its length removed}

In Fig. 7 all six normal and shear stresses are plotted for this case to accurately represent the stress state. It is evident in Fig. 7 that the cylindrical model cannot be used as a simplification for the bone geometry when three-quarters of the bone has been resected. The reduction in stress is due to the increasing cross-section of the bone at the lesser trochanter. The stresses measured in bending (Fig. 7a,b) and axial (Fig. 7c,d) loading are less than one-third of the stresses measured for the femur with one half removed (Fig. 6), and the stress measured in torsion (Fig. $7 e, f)$ is less than half, partially due to the larger diameter chosen for the implant (18mm rather than $14 \mathrm{~mm}$ ). The implant has a larger diameter in order to increase the amount of cortical bone contact and to allow for the shorter contact length $(50 \mathrm{~mm})$. The largest stress concentrations of about 10MPa are found at the distal end of the bone under bending and torsional load (Fig. 7a,e). Torsion and bending in particular generate high stresses in the cancellous bone, which must be taken into account for predicting safe load limits, as the cancellous bone has a lower strength than cortical bone. 


\section{Discussion}

The stress transferred to a femur from a directly attached implant under normal walking loads was calculated for three different resection levels. The femur of an amputee with an ITAP implant that does not extend proximal to the diaphysis can be modelled using a cylindrical bone containing the implant to calculate the stress distribution. This method has been used previously by $\mathrm{Xu}$ et al. ${ }^{13}$ and lesaka et $\mathrm{al}^{30}$. The stresses in bending and for an axial load match those for the full $3 \mathrm{D}$ anatomical model when the outer diameter of the cylindrical model is chosen as the thickness of the bone in the medial-lateral direction, as measured from a radiograph. For torsional loads this underestimates the stress levels adjacent to the implant and a 10\% smaller outer bone diameter should be chosen. Results show lower stress levels in the bone adjacent to the implant (stress shielding) and stress concentrations at the distal and proximal ends of the implanted length of the stem. The stress level and stress concentration due to bending loads were found to be significantly larger than those due to axial loading.

Xu et al. ${ }^{14}$ used FEA to analyse an intramedullary femoral implant for attachment of a prosthetic limb under typical walking loads and calculated the maximum von Mises stress in the bone near the implant tip for an amputee with a relatively long femur (equivalent to the 'quarter resected' model in this study) to be $38 \mathrm{MPa}$ and $41 \mathrm{MPa}$ for implants of $21.5 \mathrm{~mm}$ and $22 \mathrm{~mm}$ diameter. These values are comparable to the $35 \mathrm{MPa}$ von Mises stress at the implant tip found in this study. The von Mises stress at the distal end of the bone in the research by Xu el al. was $18 \mathrm{MPa}$ and $14 \mathrm{MPa}$, whereas a value of $17 \mathrm{MPa}$ was found in this study. These results are not directly comparable because the implant geometries are different, but the similar values indicate that the two techniques of prosthetic attachment lead to similar stress state and concentration in the bone.

There is a stress concentration on the stem/bone interface at the distal end of the resected bone for all models, as found by Xu et al. ${ }^{13}$ and Stephenson and Seedhom ${ }^{26}$. One way to avoid the risks associated with the distal stress concentration is to include a collar in the implant design which abuts the end of the bone. Fig. 5 shows the axial stress on the inside of the cortical bone in bending when the implant includes a collar that is osseointegrated (perfect contact conditions). The stress is evenly distributed across the distal end of the bone by the implant collar without any significant stress concentration, and this is also seen in axial loading and torsion. Inclusion of a distal collar constitutes a recommended design feature for the ITAP implant.

A fail-safe device situated between the external prosthesis and the bone anchor can prevent damage to the femur from excessive loading. A number of factors influence the loads at which the failsafe device should be set in order to protect the bone. The bending moments increase with the increasing lever arm of the point of load application (the knee). For the implant tip located in the femur shaft (diaphysis) this results in a higher stress concentration at the proximal end of the implant (Fig. 3). A possible reduction of the stress concentration was shown by placing the implant tip in the epiphysis to encounter a larger second moment of area (Fig. 6). An immediate 
conclusion might be that an implant that terminates in the epiphysis of the femur is preferable. However, it must be noted that the implant is then in contact with cancellous bone with lower strength $^{31}$. Another influence of the bone resection level on the failsafe device settings is for a very short residual bone, where the interface shear stresses for torsional loading can become critical due to the decreased length of the implant-cortical bone contact. An increase in risk for higher resection levels can be predicted, which would necessitate the use of a different implant design to facilitate the transmission of torsional loads to the bone. This assessment is supported by a retrospective study of custom-made prostheses for the replacement of bone in cancer patients by Unwin et al. ${ }^{32}$, in which it was found that the higher the level of femoral resection, the greater the probability of aseptic loosening.

The calculations in this study are based on bone of normal strength. However the femoral bone of a transfemoral amputee who has used socket prostheses or no prosthesis at all for a period of time has been found to be significantly less dense and of smaller diameter than normal bone ${ }^{29}$. It is anticipated that an ITAP user's bone will increase in density and volume as the prosthesis is loaded. Further studies would be necessary to monitor the development of the bone in users to measure the rate of increase in bone density. The femur models in this study have been represented using perfect integration between the bone and implant which is unlikely to occur in the clinical setting and would have to be taken into account. Gradual loading of the implant is required to prepare the bone for weight bearing. Proposals have been made for strengthening the bone, including gradual increase in axial load bearing using a set of scales ${ }^{2}$. More recently ${ }^{33}$ an investigation into the stress in bone around the implant for the OPRA system has concluded that gradual axial loading is insufficient to strengthen the bone in preparation for the high loads experienced near the implant tip and the distal end of the bone, and that bending and torsional loading should be prescribed in the rehabilitation programme. This is in line with the findings presented here where the most significant stress concentration was found under a bending load at the proximal end of the implant.

An implant that is stiffer than the bone 'shields' the bone from stress, as evidenced by the lower stress adjacent to the implant. Blunn et al. ${ }^{8}$ conducted FEA on cementless femoral implants for segmental bone tumour prostheses. They found that by reducing the HA coated portion of the implant to one-third, the region of bone under very low stress as a result of the loading of the implant was reduced. Radiographs of the femur of amputees in the OPRA programme were assessed over seven years by $\mathrm{Xu}$ and Robinson ${ }^{34}$ and the femur was found to narrow considerably at the distal end exposing the intramedually stem which was once enclosed with cortical bone whilst at the proximal end of the implant the cortical thickness increased. The bone remodelling changes seen around implants fixed into the diaphysis are different to those around a hip stem. Stress shielding around a hip stem results in loss of bone density whilst around an intramedullary stem fixed into the diaphysis cortical bone is removed. 


\section{Conclusions}

Anatomical finite element models of the femur were created for different levels of bone resection with osseointegrated ITAP implants. On the stem/bone interface stress concentrations are seen at the resected, distal end and adjacent to the implant tip, with the highest stress due to bending load. A cylindrical model of bone and implant can be used to estimate the stresses due to bending and axial loads in a long residual bone (implant tip distal to lesser trochanter) for a particular amputee by taking the thickness of the bone from a radiograph in the medial-lateral direction. For an amputee with a short residual limb, the implant is in contact with weaker cancellous bone and it is recommended that an amputee not be fitted with a straight implant as modelled here but a design that facilitates transmission of the stress to the cortical bone.

\section{Acknowledgements}

Competing interests: L. Newcombe and M. Dewar hold a patent for prosthetic limb attachment.

Funding: L. Newcombe acknowledges part-funding for her PhD studentship from Stanmore Implants. The company did not specifically sponsor this study and had no involvement in it.

Ethical approval: Not required

\section{References}

[1] Zhang M, Turner-Smith AR, Roberts VC, Tanner A. Frictional action at lower limb prosthetic socket interface. Med Eng Phys 1996, 18(3), 207-14.

[2] Sullivan J, Uden M, Robinson KP, Sooriakumaran S. Rehabilitation of the Trans-Femoral Amputee with an Osseointegrated Prosthesis: the United Kingdom Experience. Prost and Orth Int 2003, 27(2), 114-120.

[3] Adell R, Eriksson B, Lekholm U, Brånemark PI, Jemt T. Long-term follow-up study of osseointegrated implants in the treatment of totally edentulous jaws. Int J Oral Maxillofac Implants 1990, 5, 347-59.

[4] Brånemark PI, Hansson BO, Adell R, Breine U, Lindstrom J, Hallen O, Ohman A. Osseointegrated implants in the treatment of the edentulous jaw. Experience from a 10-year period. Scand J Plast Reconstr Surg Suppl 1977, 16, 7-132.

[5] Albrektsson T, Jansson T, Lekholm U. Osseointegrated dental implants. Dent Clin North Am 1986, 30(1), 151-74.

[6] Aschoff HH, Juhnke DL. Evaluation of 10 years experience with endo-exo femur prostheses - background, data and results. Z Orthop Unfall 2012, 150(6), 607-14

[7] Nebergall A, Bragdon C, Antonellis A, Karrholm J, Branemark R, Berlin O, Malchau H. Stable fixation of an osseointegrated implant system for above-the-knee amputees. Acta Orthop 2012 83(2), 121-8. 
[8] Blunn GW, Briggs TW, Cannon SR, Walker PS, Unwin PS, Culligan S, Cobb JP. Cementless fixation for primary segmental bone tumor endoprostheses. Clin Orthop Relat Res 2000, 372 , 223-30.

[9] Prendergast PJ. Finite element models in tissue mechanics and orthopaedic implant design. Clin Biomech 1997, 12(6), 343-66.

[10] Shultz TR, Blaha JD, Gruen TA, Norman TL. Cortical bone viscoelasticity and fixation strength of press-fit femoral stems: finite element model. J Biomech Eng 2006, 128(1), 7-12.

[11] Stephenson P. Direct skeletal fixation of an artificial limb for transfemoral amputees. A biomechanical evaluation, PhD thesis, University of Leeds, 1999.

[12] Zheng L, Luo J, Wang X, Chen J, Gu Z, Zhang X. 3D finite element analysis of bone stress around distally osseointegrated implant for artificial limb attachment [article in Chinese]. Sheng Wu Yi Xue Gong Cheng Xue Za Zhi 2007, 24(3), 554-7

[13] Xu W, Crocombe AD, Hughes SC. Finite element analysis of bone stress and strain around a distal osseointegrated implant for prosthetic limb attachment. Proc Inst Mech Eng $[\mathrm{H}] 2000$, 214, 595-602.

[14] Xu W, Xu DH, Crocombe AD Three-dimensional finite element stress and strain analysis of a transfemoral osseointegration implant. Proc Inst Mech Eng [H]. 2006, 220, 661-70

[15] Tomaszewski PK, Verdonschot N, Bulstra SK, Verkerke GJ. A comparative finite-element analysis of bone failure and load transfer of osseointegrated prostheses fixations. Ann.Biomed.Eng 2010, 38(7), 2418-27.

[16] Helgason B, Palsson H, Runarsson TP, Frossard L, Viceconti M. Risk of failure during gait for direct skeletal attachment of a femoral prosthesis: A finite element study. Med Eng Phys 2009, 31(5), 595-600.

[17] Tomaszewski PK, van Diest M, Bulstra SK, Verdonschot N, Verkerke GJ. Numerical analysis of an osseointegrated prosthesis fixation with reduced bone failure risk and periprosthetic bone loss. J Biomech 2012 45(11), 1875-80.

[18] Pendegrass CJ, Goodship AE, Price JS, Blunn GW. Nature's answer to breaching the skin barrier: an innovative development for amputees. J Anat. 2006, 209(1), 59-67.

[19] Taddei F, Schileo E, Helgason B, Cristofolini L, Viceconti M. The material mapping strategy influences the accuracy of CT-based finite element models of bones: An evaluation against experimental meausurements. Med Eng Phys 2007 29(9), 973-9.

[20] Keyak JH, Kaneko TS, Tehranzadeh J, Skinner HB. Predicting proximal femoral strength using structural engineering models. Clin Orthop Relat Res 2005 437, 219-28.

[21] Taylor WR, Roland E, Ploeg H, Hertig D, Klabunde R, Warner MD, Hobatho MC, Rakotomanana L, Clift SE. Determination of orthotropic bone elastic constants using FEA and modal analysis. J Biomech 2002, 35(6), 767-73. 
[22] Rho JY, Hobatho MC, Ashman RB. Relations of mechanical properties to density and CT numbers in human bone. Med Eng Phys 1995, 17(5), 347-55.

[23] Wolff J. The law of bone remodelling / Julius Wolff ; translated by P.G.J. Maquet and R. Furlong. Berlin ; New York : Springer-Verlag , 1986.

[24] Strait DS, Wang Q, Dechow PC, Ross CF, Richmond BG, Spencer MA, Patel BA. Modeling elastic properties in finite-element analysis: how much precision is needed to produce an accurate model? Anat Rec A Discov Mol Cell Evol Biol 2005, 283(2), 275-87.

[25] Lee WCC, Frossard LA, Hagberg K, Haggstrom E, Brånemark R, Evans JH, Pearcy MJ. Kinetics of transfemoral amputees with osseointegrated fixation performing common activities of daily living. Clin Biomech 2007, 22(6), 665-73.

[26] Stephenson P, Seedhom BB. Estimation of forces at the interface between an artificial limb and an implant directly fixed into the femur in above-knee amputees. J Orthop Sci 2002, 7(3), 292-7.

[27] Taylor SJG, Walker PS. Forces and Moments Telemetered from Two Distal Femoral Replacements During Various Activities. J. Biomech 2001, 34(7), 839-48.

[28] Stephenson P, Seedhom BB. Cross-sectional geometry of the human femur in the mid-third region. Proc Inst Mech Eng [H] 1999, 213, 159-66.

[29] Dhert WJA, Thomsen P, Blomgren AK, Esposito M, Ericson LE, Verbout AJ. Integration of press-fit implants in cortical bone: a study on interface kinetics. J Biomed Mater Res 1998, 41(4), 574-83.

[30] lesaka K, Kummer FJ, Di Cesare PE. Stress risers between two ipsilateral intramedullary stems: a finite-element and biomechanical analysis J. Arthroplasty 2005, 20(3), 386-91

[31] Rho,JY, Hobatho,MC, and Ashman,RB. Relations of mechanical properties to density and CT numbers in human bone. Med. Eng Phys. 1995,17(5), 347-355.

[32] Unwin PS, Cannon SR, Grimer RJ, Kemp HBS, Sneath RS, Walker PS. Aseptic loosening in cemented custom-made prosthetic replacements for bone tumours of the lower limb. J Bone Joint Surg Br. 1996, 78B(1), 5-13

[33] Lee WCC, Doocey JM, Brånemark R, Adam CJ, Evans JH, Pearcy MJ, Frossard LA. FE stress analysis of the interface between the bone and an osseointegrated implant for amputees--implications to refine the rehabilitation program. Clin Biomech.2008, 23(10), 124350.

[34] Xu,W. and Robinson,K. X-ray image review of the bone remodelling around an osseointegrated trans-femoral implant and a finite element simulation case study. Ann.Biomed.Eng 2008, 36(3), 435-443. 


\section{Figure and Table Captions}

Figure 1. Medial-lateral sections of femur models showing material distribution and cross-sections at locations 1, 2 and 3: (a) quarter of the femur resected, (b) half of the femur resected, (c) half of the femur resected, anterior-posterior view showing curvature of bone, (d) three-quarters of the femur resected; dotted outlines show implant geometry and alignment; arrows indicate loading location and directions.

Figure 2. Cylindrical bone model for: (a) implant, cylindrical bone, and loading location and directions shown, (b) cross-sectional view with dimensions for quarter of bone resected indicated.

Figure 3. Stresses calculated in the femur with quarter of bone removed (implanted length 160 $\mathrm{mm}$ ): a) bending, c) axial, e) torsional load on the stem/bone interface; b) bending, d) axial, f) torsional load on the outer bone surface; node paths recorded include the highest stress on the inside or outside of the bone; solid lines: anatomical FEA model; dotted lines: cylindrical model with outer diameter $26 \mathrm{~mm}$.

Figure 4. Shear stresses in femur with quarter of bone removed under torsional load; a) stem/bone interface; b) outer bone surface; anatomical model (solid line), cylindrical model with outer diameter 23mm (dotted line); tangential/axial stress (dark line), radial/tangential stress (feint line).

Figure 5. Axial stress on the stem/bone interface with and without an implant collar; femur with quarter of bone removed under bending load.

Figure 6. Stresses calculated in the femur with half of bone removed (implanted length $140 \mathrm{~mm}$ ): a) bending; c) axial, e) torsional load on the stem/bone interface; b) bending; d) axial; f) torsional load on the outer bone surface; node paths recorded include the highest stress on the inside or outside of the bone; solid lines: anatomical FEA model; dotted lines: cylindrical model with outer diameter $26 \mathrm{~mm}$.

Figure 7. Stresses calculated in the femur with three quarters removed (implanted length $50 \mathrm{~mm}$ ): a) bending; c) axial, e) torsional load on the stem/bone interface; b) bending; d) axial; f) torsional load on the outer bone surface; node paths recorded include the highest stress on the inside or outside of the bone. Marks (I) and (II) show the length of the implant in contact with bone, and (III) and (IV) show the location of the lesser trochanter.

Table 1. Material properties for cortical and cancellous bone used for the finite element model. 
Table 2. Three methods for calculating the appropriate outer diameter of cylindrical model for estimating the stresses in the femur of an amputee with a long residual limb; implant diameter 14 $\mathrm{mm}$. 

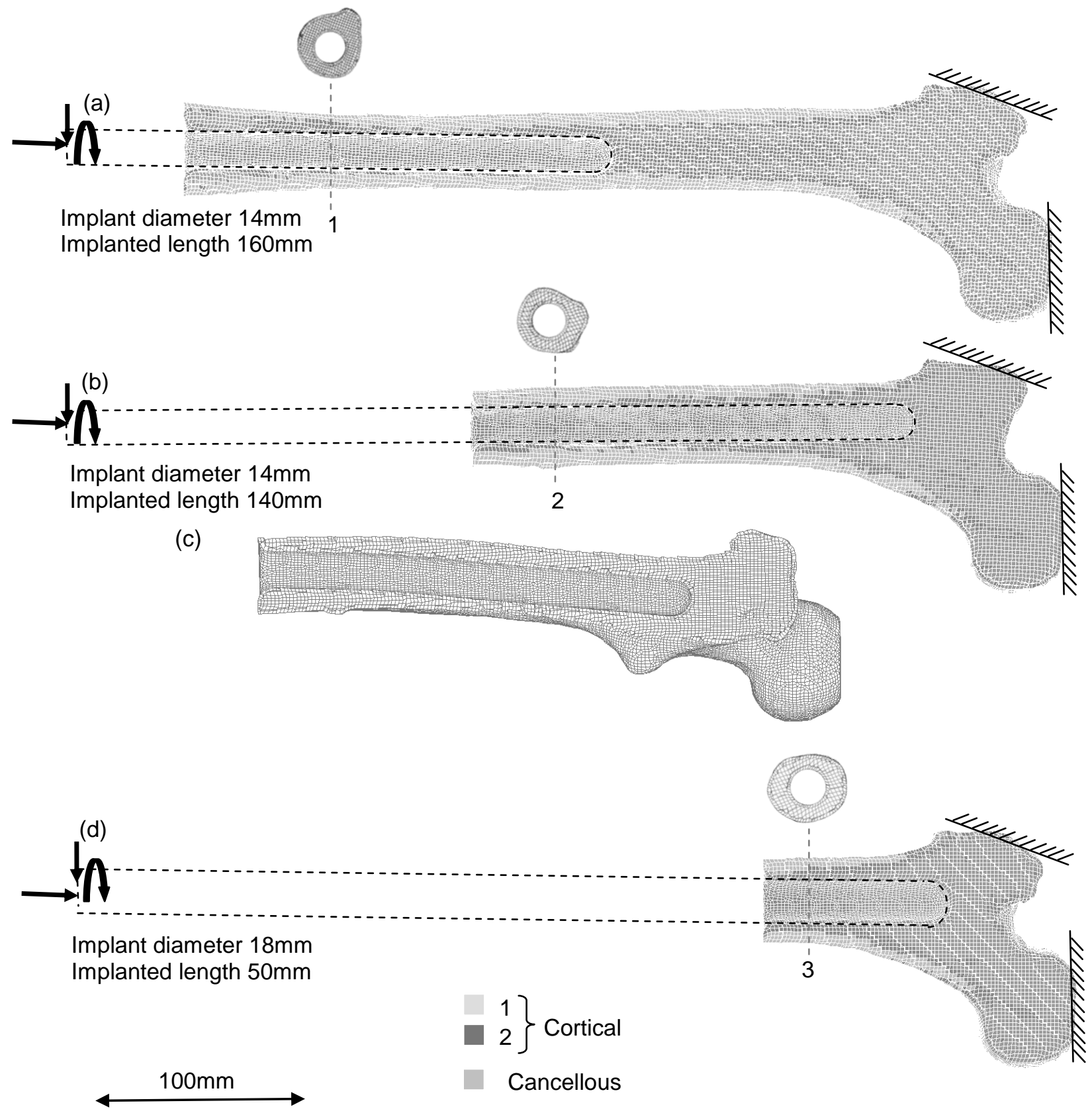


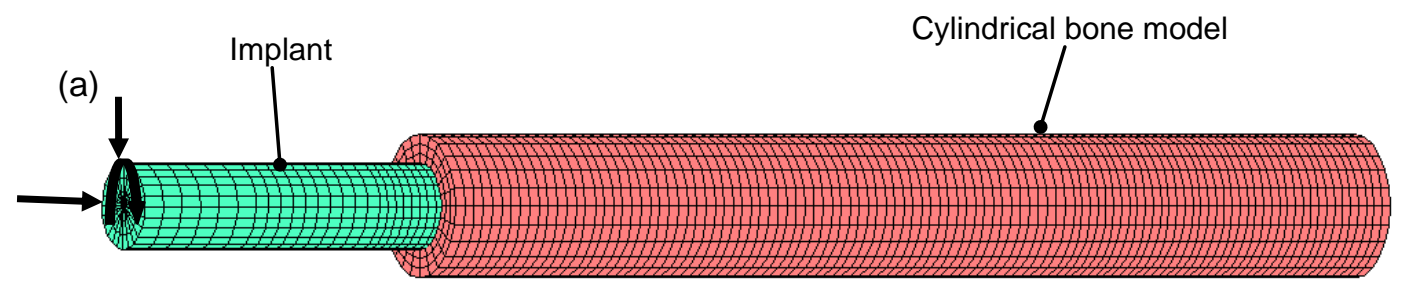

(b)

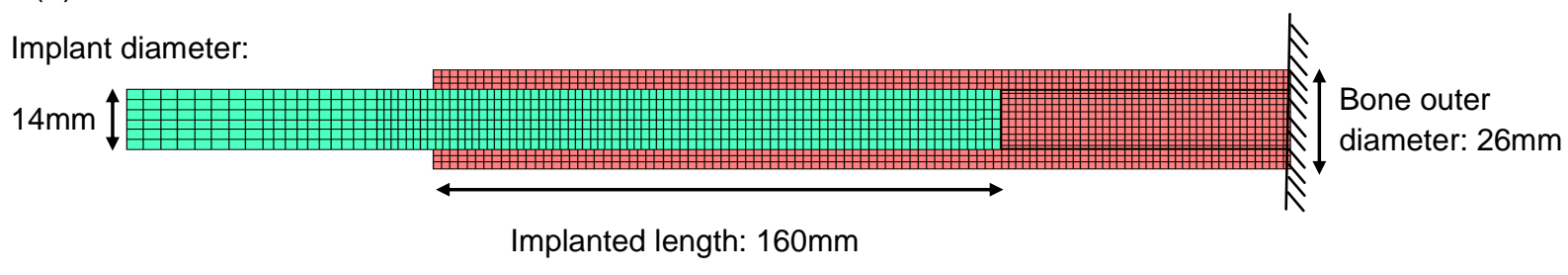




\section{Bending Load}

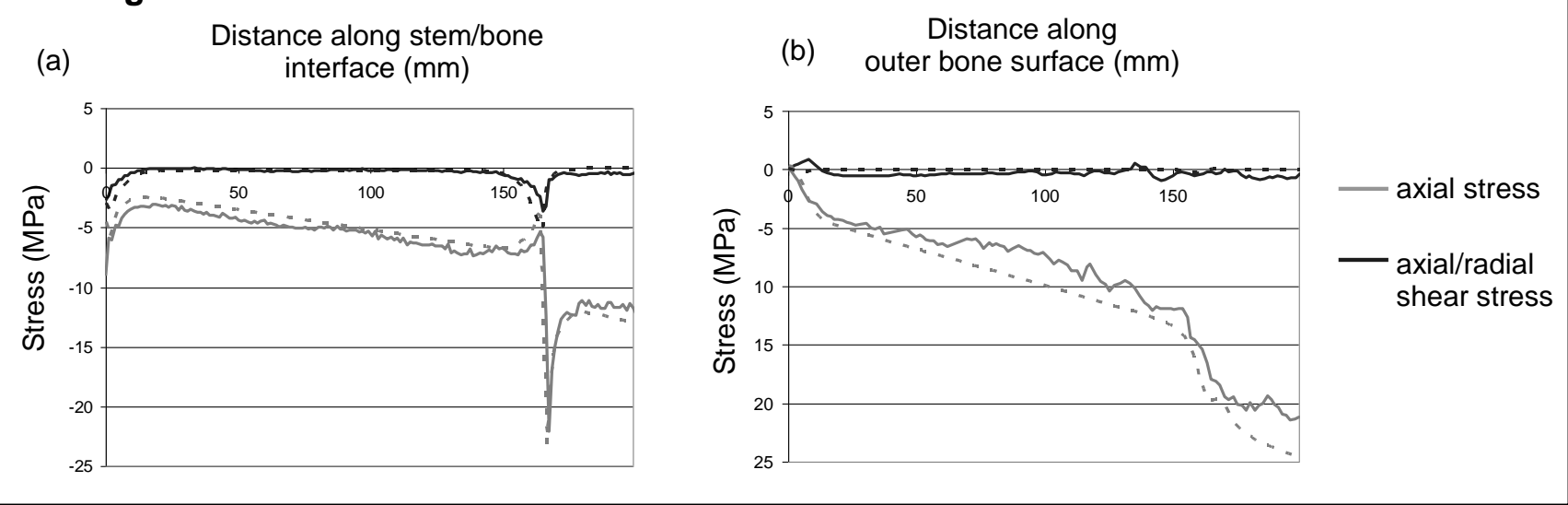

\section{Axial Load}

(c)

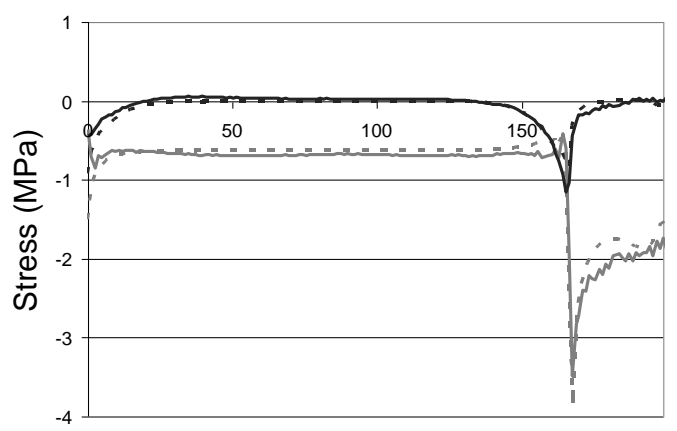

Distance along

(d) outer bone surface $(\mathrm{mm})$

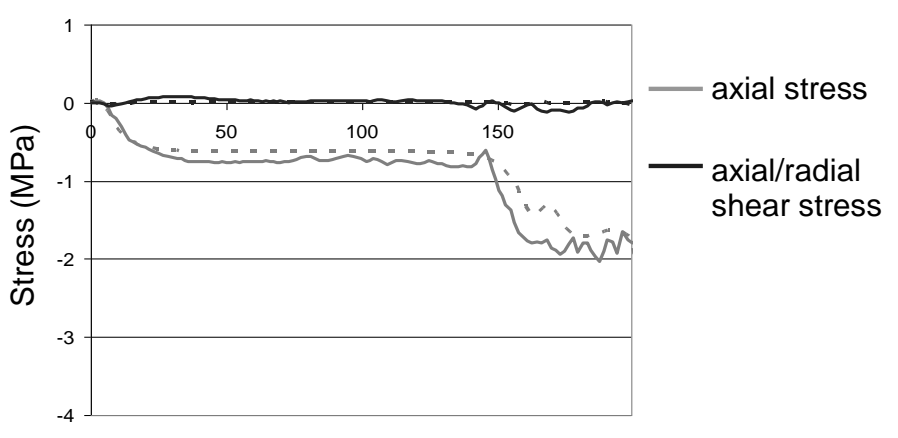

\section{Torsional Load}

(e) Distance along stem/bone

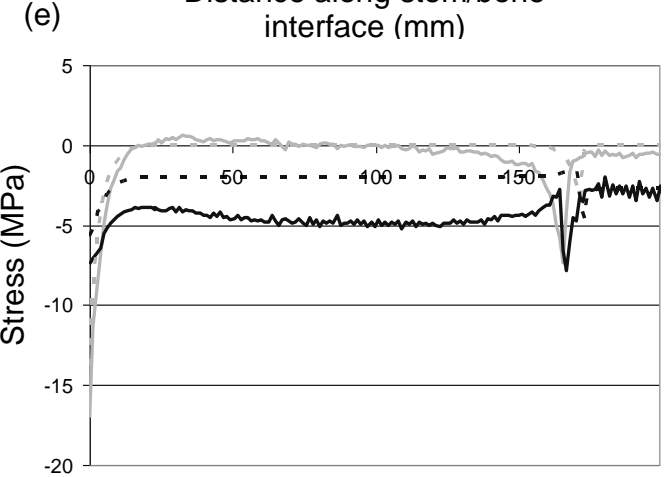

Distance along

(f) outer bone surface $(\mathrm{mm})$

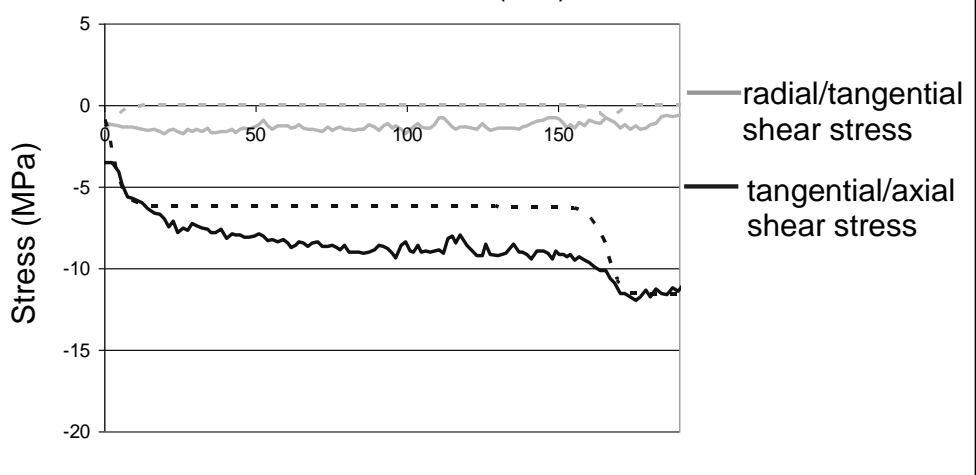


(a)

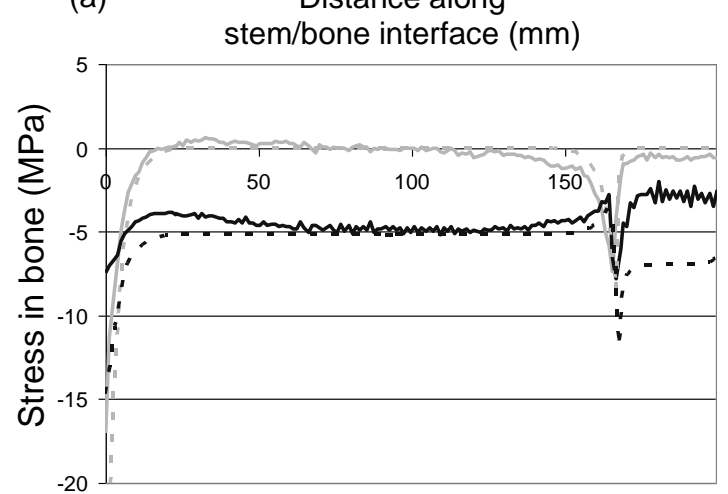

(b)

Distance along outer bone surface ( $\mathrm{mm}$ )

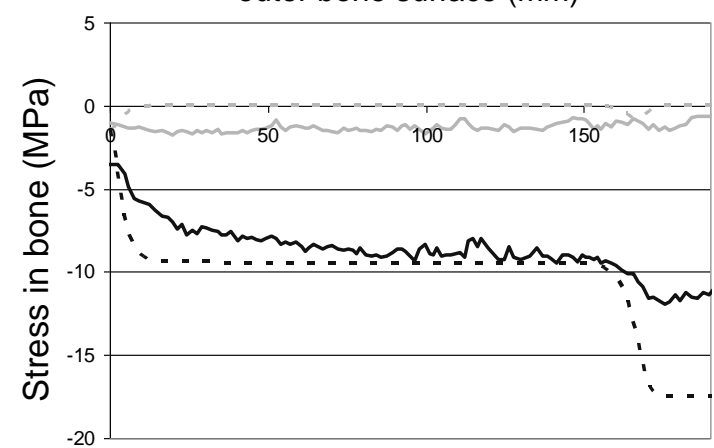


Distance along stem/bone interface $(\mathrm{mm})$

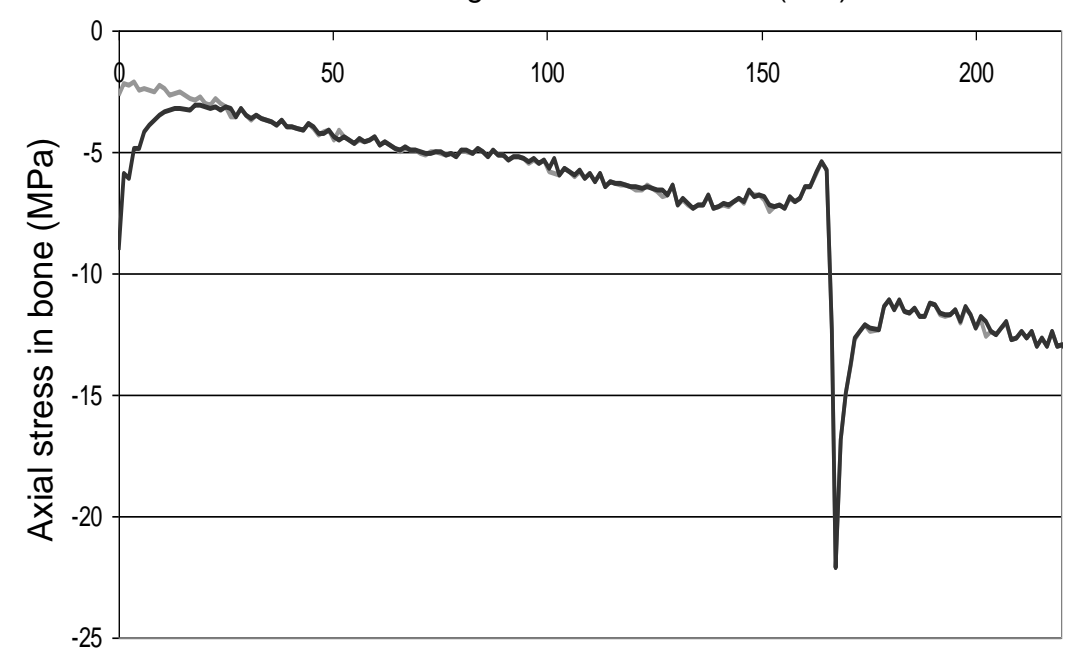

- Collared implant

- Implant without collar 


\section{Bending Load}

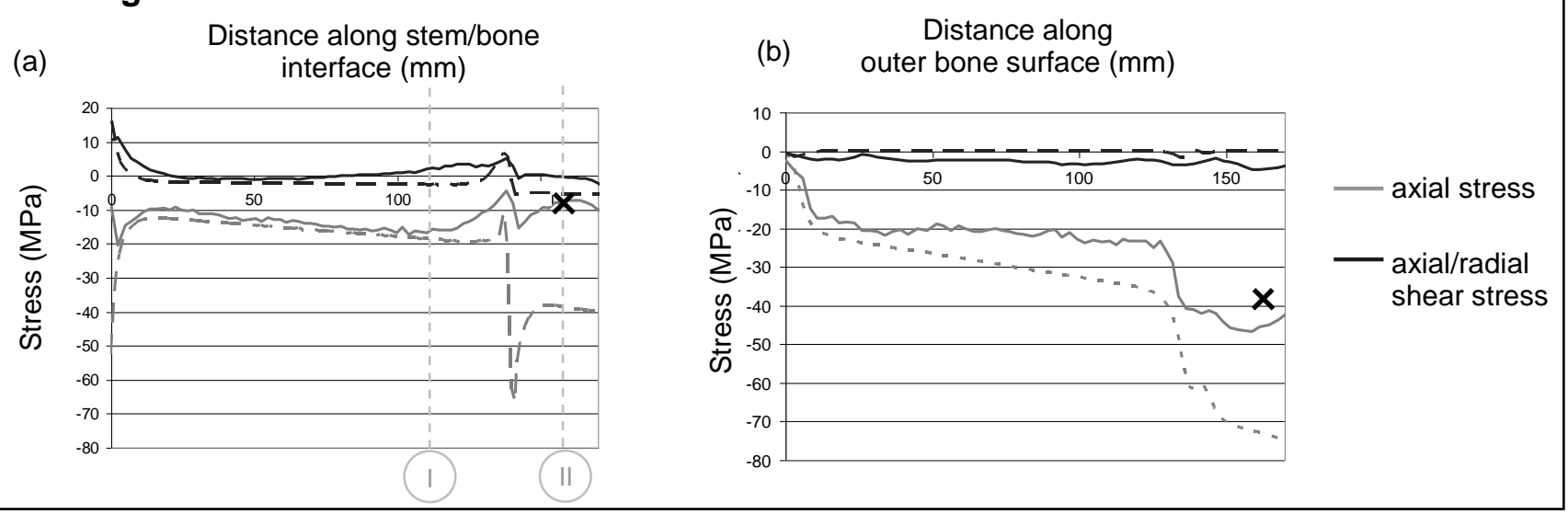

\section{Axial Load}

Distance along stem/bone

(c)

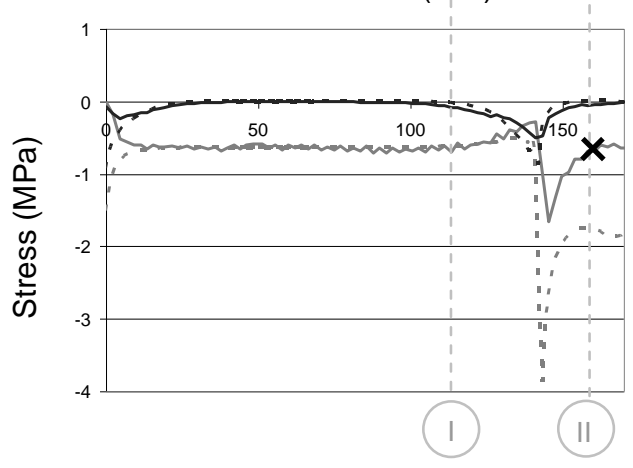

Distance along

(d) outer bone surface ( $\mathrm{mm}$ )

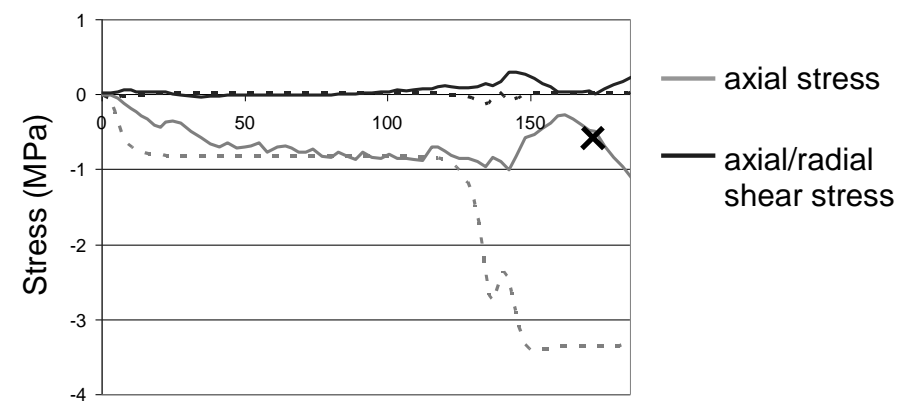

\section{Torsional Load}

(e) Distance along stem/bone

(e) interface $(\mathrm{mm})$

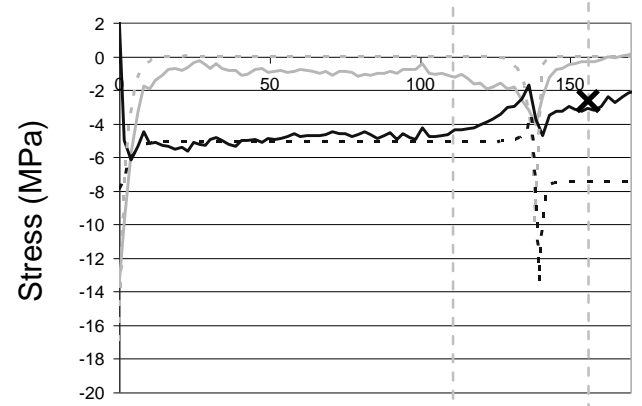

Distance along

(f) outer bone surface $(\mathrm{mm})$

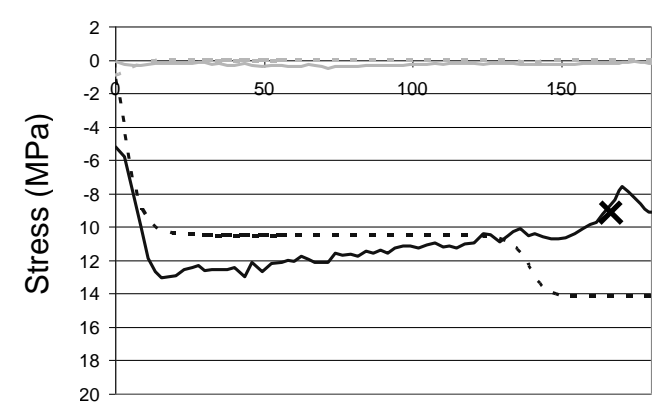

—radial/tangential shear stress

- tangential/axial shear stress 


\section{Bending Load}
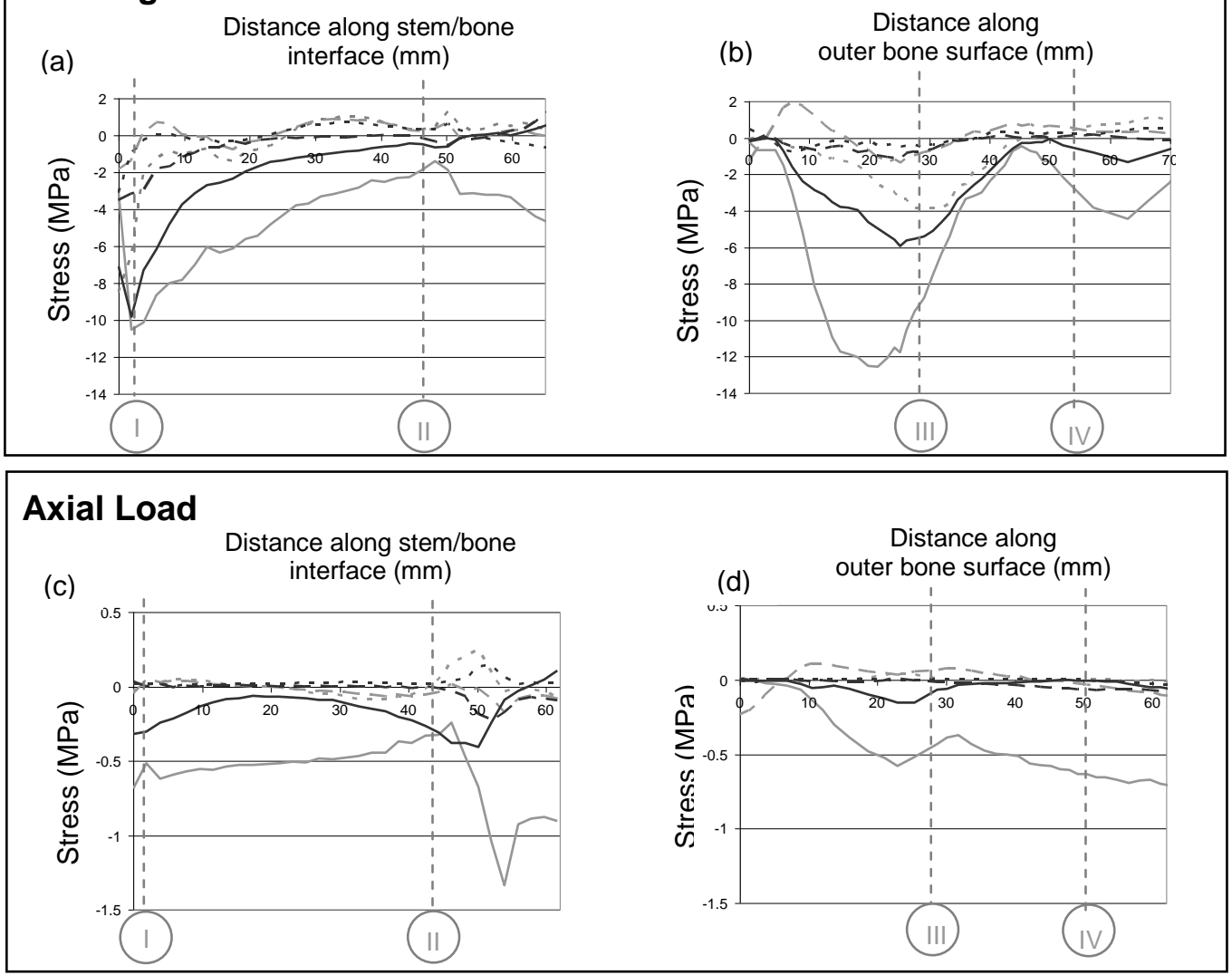

\section{Torsional Load}

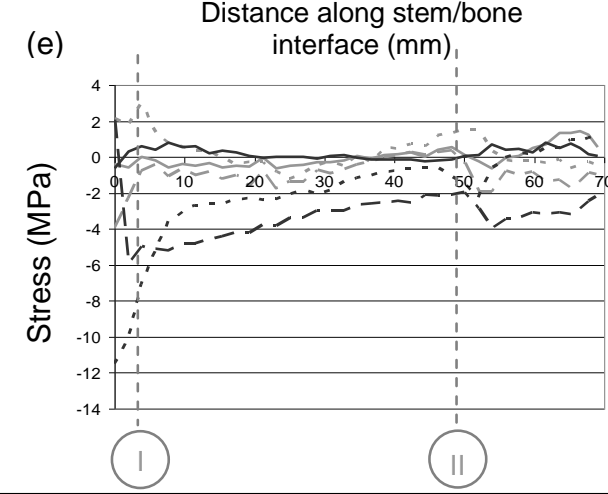

Distance along

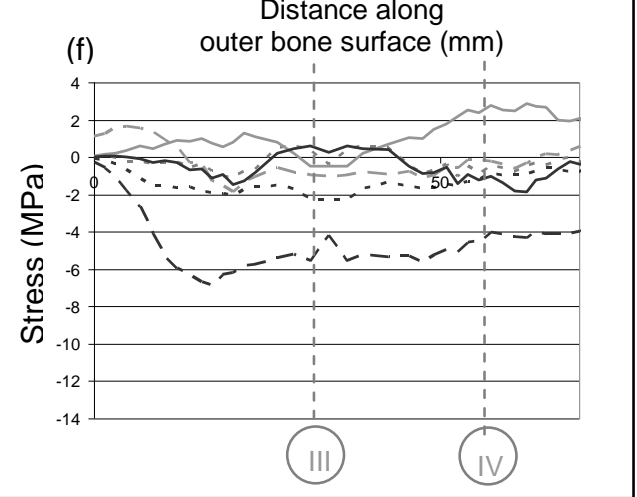

radial

\section{Anterior view}

Medial view

$$
\text { (III) (IV) }
$$

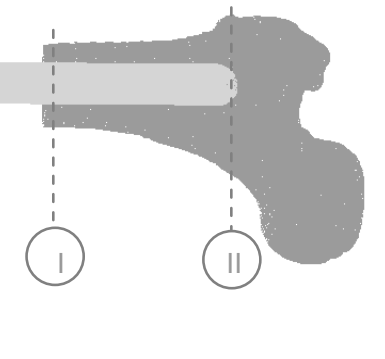

-- tangential

—axial

radial/ tangential shear

- - . tangential/ axial shear axial/radial shear 


\begin{tabular}{|c|c|c|c|c|c|c|c|c|}
\hline & & & Youns & nodulus & $\mathrm{Pa}$ & & & Shear \\
\hline & ection & & & ial num & & & 有 & (GPa) \\
\hline & & 1 & 2 & 3 & 4 & 5 & & \\
\hline & axial & 12.263 & 11.413 & & & & 0.376 & 4.530 \\
\hline $\begin{array}{l}\text { Cortical } \\
\text { bone }\end{array}$ & $\begin{array}{l}\text { transverse } \\
\text { radial }\end{array}$ & 12.508 & 11.743 & & & & 0235 & 6230 \\
\hline & $\begin{array}{l}\text { transverse } \\
\text { circumfererential }\end{array}$ & 19.548 & 18.358 & & & & 0.235 & 0.230 \\
\hline & axial & & & 7.413 & 4.539 & 1.757 & 0.376 & 4.530 \\
\hline Cancellous & $\begin{array}{l}\text { transverse } \\
\text { medial-lateral }\end{array}$ & & & 8.143 & 5.191 & 0.98 & & \\
\hline & $\begin{array}{l}\text { transverse } \\
\text { anterior- } \\
\text { posterior }\end{array}$ & & & 12.758 & 5.21 & 0.704 & 0.235 & 6.230 \\
\hline
\end{tabular}




\begin{tabular}{|l|l|l|}
\hline \multicolumn{2}{|c|}{} & Cynatomical Model \\
\hline
\end{tabular}

